\title{
Spatial representation of overlearned arbitrary visuomotor associations
}

\author{
Meike J. Grol • Ivan Toni • Mireille Lock • \\ Frans A. J. Verstraten
}

Received: 11 January 2008 / Accepted: 6 November 2008 / Published online: 2 December 2008

(C) The Author(s) 2008. This article is published with open access at Springerlink.com

\begin{abstract}
Our movements can be guided directly by spatial information, but also more flexibly through arbitrary rules. We have recently shown that as arbitrary visuomotor mappings became overlearned, they come to rely not only on fronto-striatal circuits, but also on the posterior parietal cortex (PPC). Since this region supports multiple reference frames for hand movements, the question arose whether overlearned visuomotor associations could come to rely on a spatial framework, similar to spatially guided movements.
\end{abstract}

Electronic supplementary material The online version of this article (doi:10.1007/s00221-008-1653-9) contains supplementary material, which is available to authorized users.

M. J. Grol

Leiden Institute for Brain and Cognition (LIBC),

Leiden University, Leiden, The Netherlands

M. J. Grol · M. Lock · F. A. J. Verstraten

Helmholtz Institute, Experimental Psychology,

Universiteit Utrecht, Utrecht, The Netherlands

M. J. Grol - I. Toni · M. Lock · F. A. J. Verstraten

F.C. Donders Centre for Cognitive Neuroimaging,

Radboud University Nijmegen, Nijmegen, The Netherlands

\section{J. Grol}

Institute for Psychological Research (LU-IPR),

Leiden University, Leiden, The Netherlands

\section{J. Grol (ه)}

Department of Radiology, Leiden University Medical Center,

Postzone C2-S, P.O. Box 9600, 2300 RC Leiden,

The Netherlands

e-mail: m.j.grol@lumc.nl

I. Toni

Nijmegen Institute for Cognition and Information,

Radboud University Nijmegen, Nijmegen,

The Netherlands
Alternatively, overlearned visuomotor associations could be non-spatial in nature. In this study we investigate the characteristics of the movement representations supporting arbitrary visuomotor mappings by assessing how performance of extensively trained arbitrary visuomotor associations depends on the effector used to provide the response. After extensive training on a set of arbitrary visuomotor associations, subjects were asked to perform the same task in one of two novel settings that varied either the spatial or the motor relationship between visual instructions and finger movements. We found that the change in spatial configuration resulted in a larger amount of interference on the performance of the original mappings than the configuration change in motor coordinates. This result suggests that the visual stimuli became arbitrarily coupled to locations in space and not directly to the finger movements. We infer that overlearned arbitrary visuomotor associations are represented in spatial coordinates, in an effector-independent framework. This result raises the possibility that the previously reported involvement of the posterior parietal cortex in overlearned visuomotor behavior reflects the transition from an arbitrary visuomotor mapping into a spatially based stimulus-location-response mapping.

Keywords Motor skill · Effector-dependent . Effector-independent · Conditional motor learning · Posterior parietal cortex $\cdot$ Visuomotor learning

\section{Introduction}

We are in constant interaction with our environment. Sensory input often guides our movements by providing spatial information like the location and size of an object we would like to pick up. These are spatially guided visuomotor 
transformations (Wise et al. 1996, Jeannerod et al. 1995). However, we can also use sensory information in more flexible ways, as when we steer a boat according to the color of a buoy. These are arbitrarily instructed movements (Wise and Murray 2000).

Several distinctions can be drawn between these two categories of sensorimotor transformations. For instance, spatially guided and arbitrarily instructed movements are supported by largely different parieto-frontal and frontostriatal circuits (Milner and Goodale 1995, Toni et al. 2001) and these two types of sensorimotor processes appear to be driven either by object affordances or by learned stimulusresponse-outcome mappings (Grezes et al. 2003, Passingham 1993). However, it remains unclear whether these are structural distinctions, or different occurrences of the same time-varying phenomenon. For instance, it has been suggested that the motor plan automatically afforded by an object or by a location might require a learning process that abstracts relevant stimuli features (Oztop et al. 2004). This observation emphasizes that even apparently direct spatial correspondences between stimuli and responses might be the result of sensorimotor associations learned by trial and errors and in which the relevant mapping is initially arbitrary. Analogously, arbitrary visuomotor associations can be trained to a degree of automaticity that makes them insensitive to reward devaluation and thus akin to habits (Shadmehr and Wise 2005; Packard and Knowlton 2002; White and McDonald 2002; Graybiel 1995). These considerations raise the issue of whether these two categories of sensorimotor transformations are computationally distinct, or whether training effects might account for some of the differences mentioned above.

We have recently shown that as arbitrary visuomotor mappings became overlearned, they come to rely not only on fronto-striatal circuits (Nixon et al. 2004) but also on the posterior parietal cortex (PPC) (Grol et al. 2006), a region well known for controlling spatially guided hand movements (Faillenot et al. 1997; Sakata et al. 1995). In PPC, both spatial (Battaglia-Mayer et al. 2000, 2001, 2007) and non-spatial (Lacquaniti et al. 1995) reference frames for hand movement have been identified and physiology showed that neurons in motor and premotor cortex could encode hand movement in either an intrinsic or extrinsic coordinate frame (Kakei et al. 2003). Accordingly, the question arises whether overlearned visuomotor associations could come to rely on a spatial framework, similar to spatially guided movements. Alternatively, it is possible that overlearned visuomotor associations are non-spatial in nature. In this scenario, the PPC involvement during overlearning arbitrary visuomotor associations (Grol et al. 2006) could reflect the ability of this region to encode nonspatial features of instructions that are relevant to the task, following extensive training (Sereno and Maunsell 1998;
Toth and Assad 2002). In this study we contrast these two possibilities.

To address this issue, we exploit experimental procedures developed in the context of motor skill learning (Keele et al. 1995; Hikosaka et al. 1995; Bapi et al. 2000). These procedures have been previously used to test whether the knowledge of learned sequences of movements is bound to the particular effector used for learning (effectordependent), or whether sensorimotor regularities can be generalized across different movements (effector-independent). Accordingly, here we used these procedures to assess whether the performance of extensively trained arbitrary visuomotor associations depends on the particular finger used to provide the response, or on the particular spatial location of the response. Furthermore, to increase the sensitivity of our test, we have used an interference protocol. This allowed us to assess differential interference effects induced by performing visuomotor associations in two novel settings.

After extensive training on a set of arbitrary visuomotor associations, subjects were asked to perform the same task in one of two novel settings that varied either the spatial or the motor relationship between visual instructions and finger movements. The rationale of this approach is that if the overlearned arbitrary associations have come to rely on a spatial mapping, then altering the spatial relationship between instructions and finger movements (while keeping the instruction-to-finger mapping constant) would produce stronger interference effects than altering the relationship between instructions and finger movements (while keeping the spatial layout of the instruction-to-movement mapping invariant).

\section{Methods}

\section{Participants}

We studied 25 right-handed volunteers (9 males, 16 females) with normal or corrected-to-normal vision. The participants' age ranged from 18 to 27 years (mean 22). Subjects gave informed consent according to the guidelines of the institutional ethics committee and were paid for their participation.

\section{Task and experimental setup}

Subjects learned, by trial and error, the correct associations between a set of four visual patterns (black and white line drawings) and four different motor responses (finger presses). Following the presentation of a visual pattern on a computer monitor, the subjects had to flex a finger of the right hand to press a button on a four-button keypad 


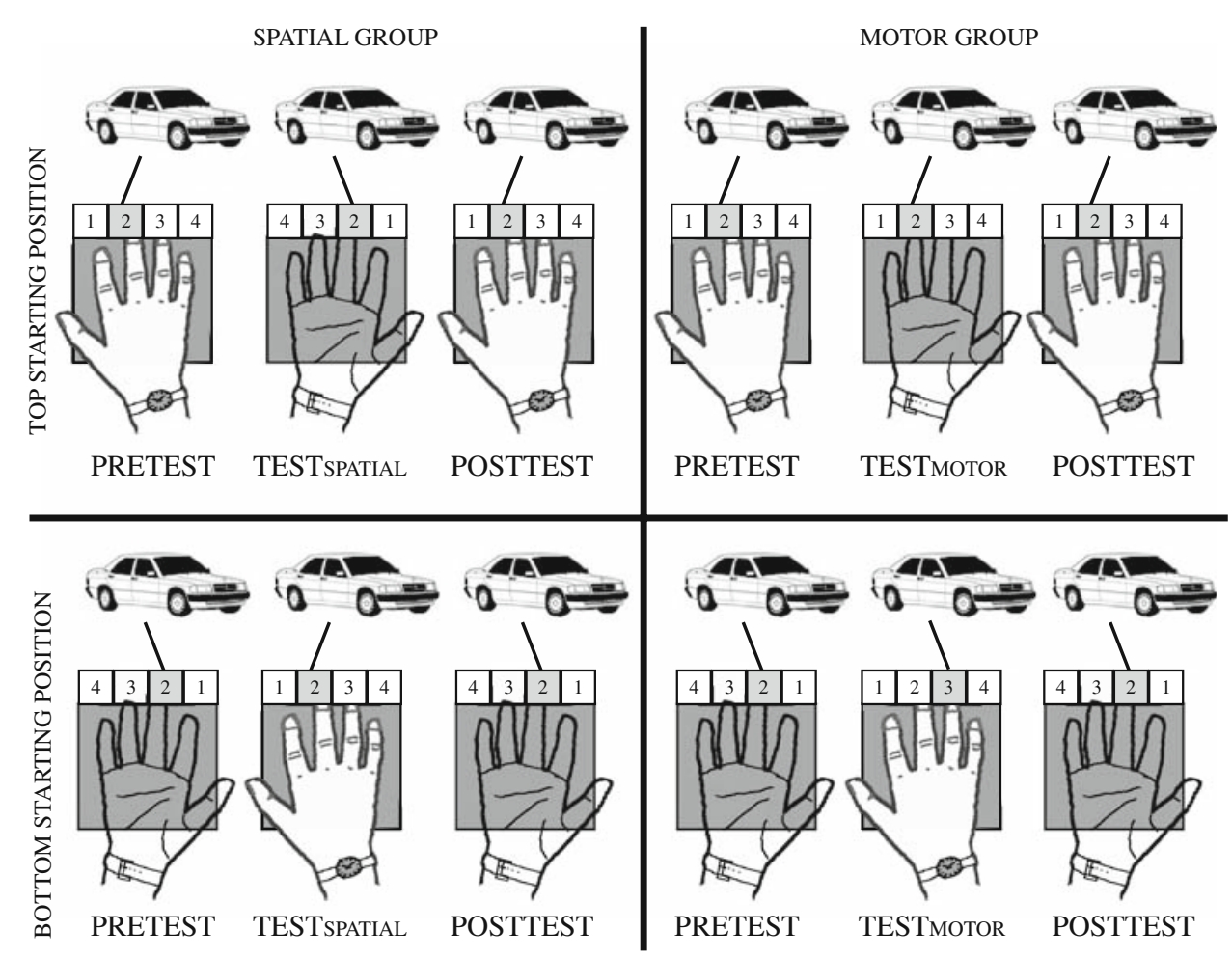

Fig. 1 Following the presentation of a visual pattern on a computer monitor, the subjects had to flex a finger of the right hand to press a button on a four-button keypad. The hand was positioned either above or below the keypad (top and bottom configuration, respectively). The subjects were divided in four groups. Half of the subjects performed the training sessions (day 1) in the top configuration (upper row), the other half in the bottom configuration (bottom row). First, during PRETEST, the subjects performed the visuomotor mappings learned on day 1 in the same configuration used during the training sessions. Second, during TEST, the subjects performed the visuomotor mappings learned on day 1 in either one of the two experimental conditions [TEST SPA- $_{\text {- }}$

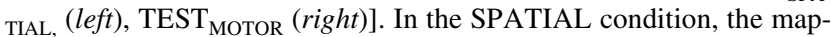
ping between the stimuli and the finger movements was the same, irrespective of the hand configuration. For instance, a car instructed a flexion of the middle finger towards the second button from the left in

(Fig. 1). Subjects positioned their index, middle, ring, and little fingers on each of four corresponding buttons of the keypad. The hand was positioned either above or below the keypad. We label these two hand configurations as "top" (Fig. 1-upper row, PRETEST) and "bottom" (Fig. 1bottom row, PRETEST), respectively. Subjects switched between these two configurations by rotating the right hand and forearm by $180^{\circ}$ along its sagittal axis, i.e. with a pronation or a supination movement around the elbow. The forearm was supported by an armrest attached to the chair on which the subject sat. The upper arm was kept in the same position in both hand-forearm configurations. In the top configuration, a cardboard prevented the vision of the hand to the subject. When the subject's hand was in the bottom configuration, the table holding the keypad prevented vision of the hand. In other words, the same amount of the top configuration (upper row, left, PRE-TEST) and a flexion of the same finger towards the third button from the left in the TEST SPATIAL. $_{\text {. }}$ This implies that the body-centered spatial position of the button associated with the car would change as a function of the hand configuration. In the MOTOR condition, the mapping between the stimuli and the buttons was the same irrespective of the hand configuration. The car here instructs to press the second button from the left on the keypad irrespective of the hand configuration (upper row, right, TEST $_{\text {MOTOR }}$ ). This implies that the finger associated with the car would change as a function of the hand configuration. In the PRE-TEST, the middle finger is associated with the car drawing, whereas in the TEST $\mathrm{MOTOR}_{\text {the ring }}$ finger is associated with the car drawing. Third, the subjects performed again the visuomotor mappings learned on day 1 (POST-TEST) in the same configuration used during the training sessions

visual information was available, irrespective of the hand configuration.

Crucially, we manipulated the spatial relation between the keypad and the fingers. There were two different experimental conditions, performed in different groups of subjects. The 'Motor' group underwent a manipulation of the relationship between the visual stimulus and the required finger movement. The 'spatial' group underwent a manipulation of the relationship between the stimulus and the button on the keypad that must be touched.

In the spatial condition, the mapping between the stimuli and the finger movements was the same, irrespective of the hand configuration. For instance, a car would instruct a middle finger flexion in both the PRETEST (Fig. 1-upper row, left) and the TEST (Fig. 1-upper row, left, TEST SPATIAL $_{\text {). }}$. This implies that the body-centered spatial position of the 
button associated with the car would change as a function of the hand configuration. Figure 1 illustrates that, in the top configuration (Fig. 1-upper row, left, PRETEST), the second button from the left is associated with the car drawing, whereas in the spatial TEST (Fig. 1-upper row, left, TEST $_{\text {SPATIAL }}$ ) the third button from the left is associated with the car drawing. In the motor condition, the mapping between the stimuli and the buttons was the same, irrespective of the hand configuration. For instance, a car would instruct to press the second button from the left on the keypad, irrespective of the hand configuration (TEST MOTOR $_{\text {). }}$. This implies that the finger associated with the car would change as a function of the hand configuration. Figure 1 (upper row, right) illustrates that, in the PRETEST the middle finger is associated with the car drawing, whereas in the motor TEST (Fig. 1, upper row, right, TEST $_{\text {MOTOR }}$ ) the ring finger is associated with the car drawing.

The visual stimuli were four black and white drawings of vehicles. The fingers were randomly assigned to the visual patterns for the different subjects. After the subjects' response, a red, green, or blue square indicated whether the movement was correct, incorrect, or exceeded a response time (RT) cutoff. The patterns were presented for $0.2 \mathrm{~s}$ every $2.2 \mathrm{~s}$ on average (range: $1.8-2.4 \mathrm{~s}$; uniform distribution), with a RT cutoff of $1.5 \mathrm{~s}$. A fixation cross was presented during the inter-stimulus intervals. Subjects were instructed to respond as fast and accurately as possible. The feedback following an incorrect response was accompanied by an auditory stimulus (duration: $50 \mathrm{~ms}$ ). Viewing distance was $80 \mathrm{~cm}$ resulting in a visual angle of about $6^{\circ}$. Stimulus control and recording of subject responses was performed with Presentation ${ }^{\circledR}$ software (Version 9.70).

\section{Procedure}

The experiment consisted of one training session and of one interference session, on two separate days. On day 1 the subjects had to learn and practice the visuomotor associations in five blocks of 300 trials. Each block was followed by a break of $2 \mathrm{~min}$. The subjects were divided in four groups. Two groups performed the training sessions starting in the top configuration (Fig. 1-upper row); the other two in the bottom configuration (Fig. 1-lower row). Three subjects that failed to perform the final block of the training session with greater than $95 \%$ correct responses were dismissed.

The interference session took place on day 2, and it was structured in three blocks. First, all four groups of subjects performed the visuomotor mappings learned on day 1 (Fig. 1, PRE-TEST, 600 trials) in the same configuration used during the training sessions ('top', 'bottom'). Second, each group from one configuration ('top' or 'bottom') performed the visuomotor mappings learned on day 1 (TEST, 600 trials) in either one of the two experimental conditions (Fig. 1, TEST MOTOR $_{\text {, TEST }}$ SPATIAL $_{\text {), counterbal- }}$ ancing starting position and tasks over the four groups. Third, all groups performed again the visuomotor mappings learned on day 1 (Fig. 1, POST-TEST, 200 trials) in the same configuration used during the training sessions.

In summary, the subjects performed 2,100 trials in the same configuration before the interference session. This number of trials was chosen on the basis of an earlier experiment (Grol et al. 2006). More precisely, in that experiment we found that after 2,550 trials the subjects had acquired a high degree of automaticity in the performance of the visuomotor associations, as indexed by a dual task procedure. In the current experiment we used considerably simpler visual patterns in order to further facilitate the overtraining of the visuomotor associations.

\section{Data analysis}

Statistical analyses were performed within the framework of the general linear model (GLM, SPSS Version 14.0). Average reaction times (RT, the time from the onset of the visual stimulus until the button press) of correct responses and error rates (ER; the percentage of incorrect responses and responses after the reaction time cut-off) were analyzed together in a repeated measures MANOVA with a withinsubject main effect of block (3 levels: PRE-TEST, TEST, POST-TEST) and the between-subject factors of condition (SPATIAL, MOTOR) and configuration (TOP, BOTTOM). Simple main effects between groups were tested with least square difference post-hoc tests (Table 1). Subjects were considered a random factor. Alpha-level was set at $P=0.05$, multivariate approach, Pillai's Trace corrected. Simple main effects between sessions were tested using post-hoc paired $t$ tests (Table 1).

\section{Results}

We analyzed the interference-session performance of 22 subjects that were able to overlearn the visuomotor associations during the training session. Figure 2 illustrates the mean RT (Fig. 2a) and ER (Fig. 2b) as a function of block and condition, pooled across configurations. It can be seen that, although subjects never practiced the task in the configuration used during the TEST block, their performance was far above chance level (25\%). This finding indicates that, overall, the overlearned visuomotor associations were largely generalized to a novel spatial or motor configuration. Nevertheless, performing the task in a novel configuration had obvious effects on performance. There were significant differences between the PRE-TEST and the 
Table 1 Posthoc tests

\begin{tabular}{|c|c|c|c|c|}
\hline & $\begin{array}{l}\text { RT: mean } \\
\text { difference }\end{array}$ & $\begin{array}{l}\text { RT: standard error } \\
\text { of the mean }\end{array}$ & $\begin{array}{l}\text { ER: mean } \\
\text { difference }(\%)\end{array}$ & $\begin{array}{l}\text { ER: standard error } \\
\text { of the mean }(\%)\end{array}$ \\
\hline \multicolumn{5}{|l|}{ LSD pairwise comparisons } \\
\hline PRE-TEST versus TEST SPATIAL $_{\text {}}$ & -94 & 22 & -4.9 & 1.3 \\
\hline PRE-TEST versus TEST $_{\text {MOTOR }}$ & -74 & 13 & -1.7 & 0.4 \\
\hline PRE-TEST versus POST-TEST SPATIAL $_{\text {PAL }}$ & -37 & 16 & -3.1 & 0.7 \\
\hline PRE-TEST versus POST-TEST MOTOR $_{1}$ & -30 & 13 & -1.5 & 0.6 \\
\hline \multicolumn{5}{|l|}{ Posthoc paired $t$ tests } \\
\hline PRE-TEST $_{\text {SPATIAL }}$ versus PRE-TEST MOTOR & 27 & 23 & 0.5 & 0.6 \\
\hline TEST $_{\text {SPATIAL }}$ versus TEST $\mathrm{T}_{\text {MOTOR }}$ & 48 & 38 & 3.7 & 1.6 \\
\hline POSTTEST spatial versus POSTTEST motor & 34 & 35 & 2.0 & 1.2 \\
\hline
\end{tabular}

Mean difference and standard error of the mean for RT and ER of separate groups and sessions. Simple main effects between groups were tested with least square difference post-hoc tests. Simple main effects between sessions were tested using post-hoc paired $t$ tests. Non-significant comparisons are printed in bold

subsequent blocks (TEST, POST-TEST; main effect of block: RT: $F(2,36)=41.882 ; P<0.001 ;$ ER: $F(2,36)=$ 19.275; $P<0.001)$. Subjects were slower and less accurate during the interference test for both conditions (Table 1: PRE-TEST vs. TEST SPATIAL $_{\text {and PRE-TEST vs. TEST }}$ MOTOR;). This finding indicates that the interference test was effective in altering the performance of the visuomotor associative task. This interference was not limited to the trials of the TEST block although the mappings during the POST-TEST were identical to those of the PRE-TEST. Subjects' performance was detrimentally influenced by the interference test for both conditions (Table 1: PRE-TEST

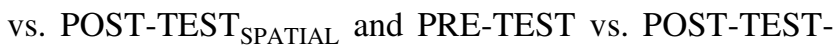
MOTOR). This finding shows that following performance of the same associations in different motor or spatial coordinates, the subjects needed to re-learn the associations in the original coordinates. Taken together, these data indicate that our experimental manipulation was successful at inducing interference effects.

The main point of this study was to test whether overlearned arbitrary visuomotor associations are represented in a spatial (effector-independent) or in a motor (effectordependent) framework. The former hypothesis would predict a stronger interference effect during the TEST SPATIAL $_{2}$ than during the TEST $\mathrm{T}_{\text {MOTOR }}$ condition. This is what we found. Subjects in the SPATIAL condition made significantly more errors during the TEST and POST-TEST blocks, as compared to the PRE-TEST block, than subjects in the MOTOR condition (interaction block $\times$ condition: ER: $F(2,36)=4.702 ; P<0.015)$. This effect was strongly driven by accuracy differences during the TEST block (Table 1: TEST SPATIAL $_{\text {vs. TEST }}$ MOTOR ;), and this was not a speed-accuracy trade-off, since there was no effect on RT (interaction block $\times$ condition: $\quad$ RT: $\quad F(2,36)=1.07$;
$P<0.35)$. In addition, there was a significant difference in the POSTTEST between subjects in the spatial group and subjects in the motor group in ER (Table 1: POSTTEST SPA- $_{\text {- }}$ TIAL $_{\text {vs. POSTTEST }}$ MOTOR). Again, no effect was found in RT. Figure $2 \mathrm{c}-\mathrm{h}$ shows the average error rate over time (in blocks of 30 trials) for all epochs. During the TEST epoch the spatial group (Fig. 2e) showed a fast learning curve in the first block, while a similar curve was absent in the motor group (Fig. 2f). The time series for the RT do not show a learning effect nor differ over groups.

We performed further controls to verify the specificity of these effects. First, we assessed whether the initial hand position (i.e. the factor configuration) influenced the subsequent interference effects, and there was no significant influence on performance (interaction block $\times$ condition $\times$ configuration: ER: $F(2,36)=0.97 ; P<0.39$; RT: $F(2,36)=$ $1.53 ; P<0.23)$. This finding indicates that the interference effects were comparable across the two groups with different initial hand positions. Second, the hand configuration had an influence of the speed of overall task performance (interaction block $\times$ configuration: RT: $F(2,36)=8.03$; $P<.001)$, a likely reflection of the greater familiarity subjects have with keyboards laying under rather than above their hands. However, this effect did not influence task accuracy (interaction block $\times$ configuration: ER: $F(2,36)=.23 ; P<0.799)$. Third, the between-conditions difference observed at TEST was not a by-product of between-groups chance variations in the PRE-TEST data, i.e., there were no significant performance differences at PRE-TEST as a function of the subsequent subdivision of the subjects pool in the SPATIAL and MOTOR sub-groups (Table 1: PRE-TEST SPATIAL $_{\text {vs. PRE-TEST }}$ MOTOR), confirming that both groups did not differ in overall performance before the interference manipulation started. 
Fig. 2 Mean reaction time (a) and error rate (b) ( \pm standard deviation) over Sessions for the SPATIAL and the MOTOR group of subjects. $\mathbf{c}-\mathbf{h}$ Mean error rate over time during training and test epochs. c Training sessions on day 1 over 15 blocks of 100 trials for all 22 subjects, $\mathbf{d}$ PRETEST epoch on day 2 over 10 blocks of 30 trials for all 22 subjects, e TEST spatial on day 2 over 10 blocks of 30 trials for 11 subjects in the spatial group, $\mathbf{f}$ TEST motor on day 2 over 10 blocks of 30 trials for 11 subjects in the motor group, $\mathbf{g}$ POSTTEST spatial on day 2 over 6 blocks of 30 trials for 11 subjects in the spatial group, $\mathbf{h}$ POSTTEST motor on day 2 over 6 blocks of 30 trials for 11 subjects in the motor group motor group (different finger, same key)

spatial group (different key, same finger)
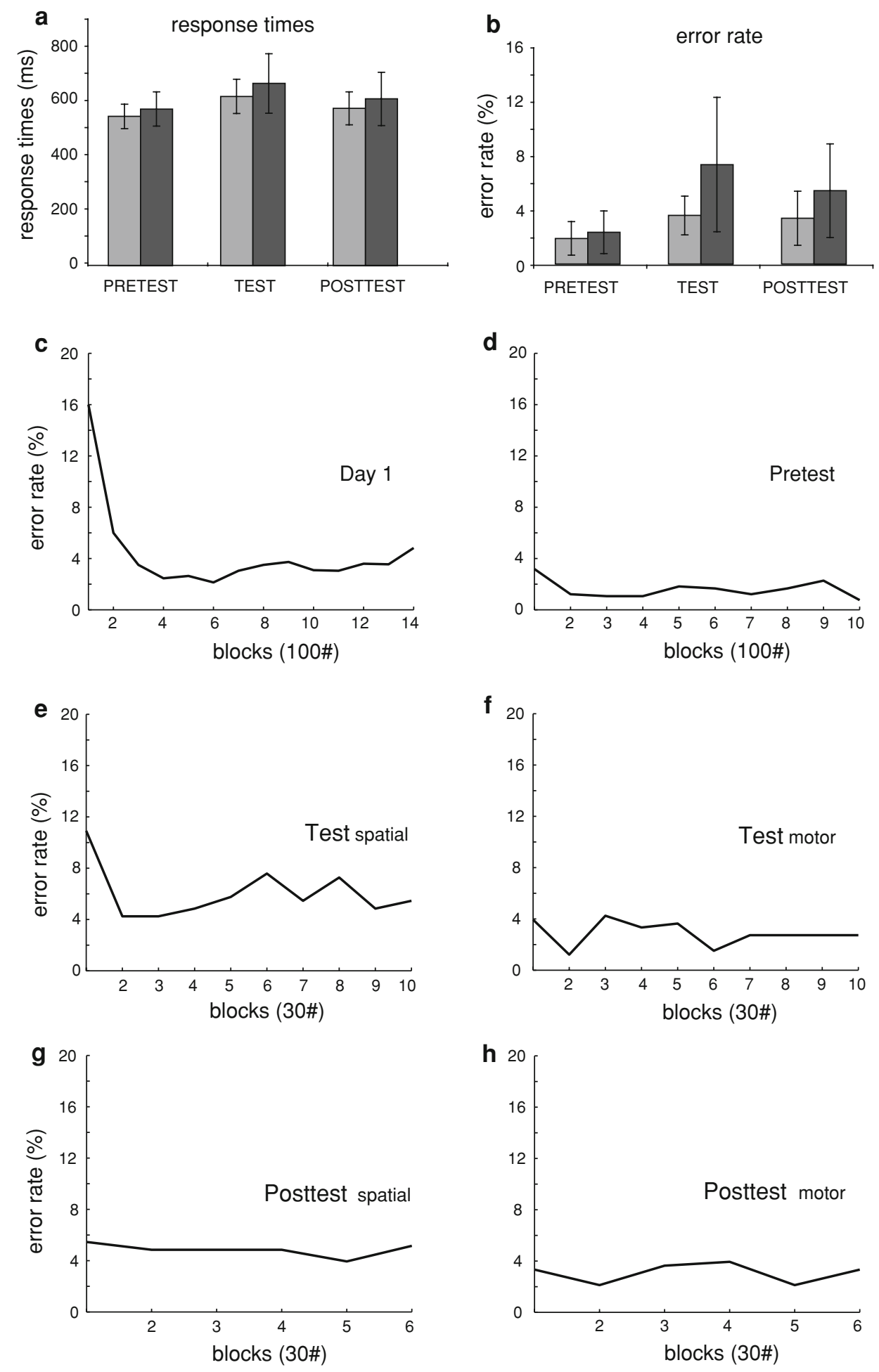

Furthermore, the timeseries of the training sessions (Fig. 2c-d) show a rapid decrease in RT and ER during the first 400-600 trials (first two training blocks, Fig. 2c), followed by a plateau (Fig. 2d), pointing to a stable performance.

\section{Discussion}

The aim of this experiment was to investigate whether overlearned arbitrary visuomotor associations are represented in a motor or in a spatial framework. After an initial 
extensive training on performance of these associations, subjects were asked to perform the same task in a novel setting that interfered with either the spatial or the motor coordinates of their performance. Following this manipulation, we also tested whether performing the visuomotor associations in the original training setting was influenced by the type of interference experienced by the subjects. We found that performance was influenced by this experimental manipulation, with stronger interference effects following spatial alterations in the relationship between visual instructions and finger movements that preserved the instruction-to-finger mapping. This result suggests that extensively trained arbitrary visuomotor associations are retrieved using a spatial framework linking visual instructions with locations in space (the buttons of the keypad). It can also be inferred that these arbitrary mappings become independent from direct couplings between stimuli and finger movements, i.e., they come to rely on an effectorindependent representation. Finally, having previously shown that under substantially identical procedural conditions, a particular portion of the PPC is involved in supporting the performance of overlearned arbitrary visuomotor associations (Grol et al. 2006), we speculate that such PPC contribution might operate according to a spatial frame of reference.

A vast body of work has addressed the issue of effectorindependent representations in the context of learning and automatization of motor skills (Wright 1990; Morton et al. 2001). For instance, finger tapping and motor sequence learning are thought to be largely independent from the effector used to produce the motor responses (Laszlo et al. 1970; Japikse et al. 2003; Taylor and Heilman 1980). Several studies have shown positive interlimb skill transfer, for example in writing from dominant to non-dominant hand (Wright 1990), ball catching (Morton et al. 2001), finger tapping (Laszlo et al. 1970), and sequential finger movements (Japikse et al. 2003, Taylor and Heilman 1980). However, other authors have argued that overlearning might generate effector-dependent representations (Jordan 1995). For instance, Thut et al. (1996) found that acquiring the skill to draw with the left hand negatively interfered with contralateral performance. It has also been suggested that both effector-dependent and effector-independent representations play a role in learning motor skills, with a relative contribution that varies as a function of the learning stage (Nakahara et al. 2001; Bapi et al. 2000; Hikosaka et al. 1999). Early in learning, changes in performance mostly consist of fast improvements in accuracy, and these changes could rely on effector-independent representations; late in learning, changes in performance mostly consist of slow improvements in speed, and these changes could rely on effector-dependent representations. This explanatory framework is supported by a functional separation in the neural correlates of these two processes (Sakai et al. 1998). Accordingly, it could be argued that our observation window was biased towards fast (effector-independent) changes, rather than towards slow (effector-dependent) changes. However, as our result might at first glance seem counterintuitive, it should be emphasized that the effects we report are not about learning of a novel motor task, but rather about the relative degree of interference evoked by two different manipulations upon extensively trained visuomotor associations. Our aim was to investigate this special situation in visuomotor behavior. Since it was shown that small changes in task requirements cause subjects to adopt different reference frames for encoding movements (Sober and Sabes 2005), these results might be highly dependent on the particular task and specific to the overlearning stage of this task.

Furthermore, it should be emphasized that motor skills and arbitrary mappings differ in a number of important aspects, and that explanatory frameworks for the former might not hold for the latter. For instance, motor skills have been studied by means of sequence learning experiments, with performance parametrized through error rates and reaction times describing the sum of incorrect sequences and the total amount of time before a complete sequence was correctly performed. These performance measures are different from those used in our experiment. In addition, motor sequences are inherently spatial, whereas in our experiment the relation between the stimuli and movements is entirely arbitrary. Motor sequences, after sufficient learning, can be performed irrespective of visual instructions, whereas in arbitrary visuomotor mapping each instruction remains necessary for selecting the correct response. Accordingly, the present findings support the observation that overlearned visuomotor associations might rely not only on the frontostriatal circuit known to be involved in arbitrary visuomotor learning (Toni and Passingham 1999; Wise and Murray 2000; Nixon et al. 2004), but also on portions of the posterior parietal cortex (Grol et al. 2006). Although fronto-striatal circuits will still be necessary to select the appropriate response, when the mappings become overlearned, the PPC might start to contribute to task performance by specifying how the response should be performed.

We suggest that once the coupling between a stimulus and the spatial location of the associated motor response has become automatic, the movement of the finger towards this location might start to resemble a spatially guided movement. We know intraparietal cells can be trained to encode the motor relevance of visual stimuli specifying arbitrary movements (Sereno and Maunsell 1998; Toth and Assad 2002) and can transform spatial information directly between different reference frames (Andersen and Buneo 2002). Primate data during visually guided reaching suggest that the PPC might form an effector-dependent 
intermediate stage transforming target location into a motor plan (Chang et al. 2008). We could therefore speculate that the cortico-cortical connections between parietal areas and premotor cortex subserve the spatial-to-motor mapping from keypad location to finger movement by translating head-centered information into a hand-centered reference frame.

\section{Conclusions}

This experiment provides empirical evidence suggesting that overlearned arbitrary visuomotor associations are represented in an effector-independent framework in spatial coordinates. Following extensive training, it appears that visual stimuli became arbitrarily coupled to locations in space and not directly to the finger movements. We speculate that during learning the increased coupling of the stimulus to its location in space might change the arbitrary mapping into a more spatially guided movement, compatible with the involvement of the posterior parietal cortex in overlearned visuomotor behavior (Grol et al. 2006).

Acknowledgment I. T. is supported by NWO (VIDI grant no. 45203-339). M. G and F. V. are supported by the Netherlands Organisation for Scientific Research (NWO-PIONIER Grant).

Open Access This article is distributed under the terms of the Creative Commons Attribution Noncommercial License which permits any noncommercial use, distribution, and reproduction in any medium, provided the original author(s) and source are credited.

\section{References}

Andersen RA, Buneo CA (2002) Intentional maps in posterior parietal cortex. Ann Rev Neurosci 25:189-220

Bapi RS, Doya K, Harner AM (2000) Evidence for effector independent and dependent representations and their differential time course of acquisition during motor sequence learning. Exp Brain Res 132:149-162

Battaglia-Mayer A, Ferraina S, Mitsuda T, Marconi B, Genovesio A, Onorati P, Lacquaniti F, Caminiti R (2000) Early coding of reaching in the parietooccipital cortex. J Neurophysiol 83:2374-2391

Battaglia-Mayer A, Ferraina S, Genovesio A, Marconi B, Squatrito S, Molinari M, Lacquaniti F, Caminiti R (2001) Eye-hand coordination during reaching. II. An analysis of the relationships between visuomanual signals in parietal cortex and parieto-frontal association projections. Cereb Cortex 11:528-544

Battaglia-Mayer A, Mascaro M, Caminiti R (2007) Temporal evolution and strength of neural activity in parietal cortex during eye and hand movements. Cereb Cortex 17:1350-1363

Chang SWC, Dickinson AR, Snyder LH (2008) Limb-specific representation for reaching in the posterior parietal cortex. J Neurosci 28:6128-6140

Faillenot I, Toni I, Decety J, Gregoire MC, Jeannerod M (1997) Visual pathways for object-oriented action and object recognition: functional anatomy with PET. Cereb Cortex 7:77-85

Graybiel AM (1995) Building action repertoires: memory and learning functions of the basal ganglia. Curr Opin Neurobiol 5:733-741
Grezes J, Tucker M, Armony J, Ellis R, Passingham RE (2003) Objects automatically potentiate action: an fMRI study of implicit processing. Eur J NeuroSci 17:2735-2740

Grol MJ, de Lange FP, Verstraten FAJ, Passingham RE, Toni I (2006) Cerebral changes during performance of overlearned arbitrary visuomotor associations. J Neurosci 26:117-125

Hikosaka O, Rand MK, Miyachi S, Miyashita K (1995) Learning of sequential movements in the monkey-process of learning and retention of memory. J Neurophysiol 74:1652-1661

Hikosaka O, Nakahara H, Rand MK, Sakai K, Lu X, Nakamura K, Miyachi S, Doya K (1999) Parallel neural networks for learning sequential procedures. Trends Neurosci 22:464-471

Japikse KC, Negash S, Howard JH Jr, Howard DV (2003) Intermanual transfer of procedural learning after extended practice of probabilistic sequences. Exp Brain Res 148:38-49

Jeannerod M, Arbib MA, Rizzolatti G, Sakata H (1995) Grasping objects: the cortical mechanisms of visuomotor transformation. Trends Neurosci 18:314-320

Jordan MI (1995) The organization of action sequences: evidence from a relearning task. J Mot Behav 27:179-192

Kakei S, Hoffman DS, Strick PL (2003) Sensorimotor transformations in cortical motor areas. Neurosci Res 46:1-10

Keele SW, Jennings P, Jones S, Caulton D, Cohen A (1995) On the modularity of sequence representation. J Mot Behav 27:17-30

Lacquaniti F, Guigon E, Bianchi L, Ferraina S, Caminiti R (1995) Representing spatial information for limb movement: role of area 5 in the monkey. Cereb Cortex 5:391-409

Laszlo JI, Baguley RA, Bairstow PJ (1970) Bilateral transfer in tapping skill in absence of peripheral information. J Mot Behav 2:261-271

Milner AD, Goodale MA (1995) The visual brain in action. Oxford University Press, Oxford

Morton SM, Lang CE, Bastian AJ (2001) Inter- and intra-limb generalization of adaptation during catching. Exp Brain Res 141:438445

Nakahara H, Doya K, Hikosaka O (2001) Parallel cortico-basal ganglia mechanisms for acquisition and execution of visuomotor sequences-a computational approach. J Cog Neurosci 13:626-647

Nixon PD, McDonald KR, Gough PM, Alexander IH, Passingham RE (2004) Cortico-basal ganglia pathways are essential for the recall of well-established visuomotor associations. Eur J NeuroSci 20:3165-3178

Oztop E, Bradley NS, Arbib MA (2004) Infant grasp learning: a computational model. Exp Brain Res 158:480-503

Packard MG, Knowlton BJ (2002) Learning and memory functions of the basal ganglia. Ann Rev Neurosci 25:563-593

Passingham RE (1993) The frontal lobes and voluntary action. Oxford University Press, Oxford

Sakai K, Hikosaka O, Miyauchi S, Takino R, Sasaki Y, Putz B (1998) Transition of brain activation from frontal to parietal areas in visuomotor sequence learning. J Neurosci 18:1827-1840

Sakata H, Taira M, Murata A, Mine S (1995) Neural mechanisms of visual guidance of hand action in the parietal cortex of the monkey. Cereb Cortex 5:429-438

Sereno AB, Maunsell JH (1998) Shape selectivity in primate lateral intraparietal cortex. Nature 395:500-503

Shadmehr R, Wise SP (2005) The computational neurobiology of reaching and pointing: a foundation for motor learning. MIT Press, Cambridge

Sober SJ, Sabes PN (2005) Flexible strategies for sensory integration during motor planning. Nat Neurosci 8:490-497

Taylor HG, Heilman KM (1980) Left-hemisphere motor dominance in righthanders. Cortex 16:587-603

Thut G, Cook ND, Regard M, Leenders KL, Halsband U, Landis T (1996) Intermanual transfer of proximal and distal motor engrams in humans. Exp Brain Res 108:321-327 
Toni I, Passingham RE (1999) Prefrontal-basal ganglia pathways are involved in the learning of arbitrary visuomotor associations: a PET study. Exp Brain Res 127:19-32

Toni I, Rushworth MF, Passingham RE (2001) Neural correlates of visuomotor associations: spatial rules compared with arbitrary rules. Exp Brain Res 141:359-369

Toth LJ, Assad JA (2002) Dynamic coding of behaviourally relevant stimuli in parietal cortex. Nature 415:165-168

White NM, McDonald RJ (2002) Multiple parallel memory systems in the brain of the rat. Neurobiol Learn Mem 77:125-184
Wise SP, Murray EA (2000) Arbitrary associations between antecedents and actions. Trends Neurosci 23:271-276

Wise SP, Murray EA, Gerfen CR (1996) The frontal cortex-basal ganglia system in primates. Crit Rev Neurobiol 10:317-356

Wright CE (1990) Generalized motor programs: reexamining claims of effector independence in writing. In: Jeannerod M (ed) Attention and performance XIII: motor representation and control. Erlbaum Associates, Hillsdale, pp 294-320 